\title{
Rule of Fives: Identifying Critical Misalignment of Strategic Choices in Organizational Design
}

\author{
Timothy Nerenz \\ University of Maryland University College-Europe, Kaiserslautern, Germany \\ Email: timothy.nerenz@faculty.umuc.edu
}

How to cite this paper: Nerenz, T. (2019) Rule of Fives: Identifying Critical Misalignment of Strategic Choices in Organizational Design. Open Journal of Business and Management, 7, 43-58. https://doi.org/10.4236/ojbm.2019.71003

Received: October 12, 2018

Accepted: November 23, 2018

Published: November 26, 2018

Copyright $\odot 2019$ by author and Scientific Research Publishing Inc. This work is licensed under the Creative Commons Attribution International License (CC BY 4.0).

http://creativecommons.org/licenses/by/4.0/

\section{(c) (i) Open Access}

\begin{abstract}
Business leaders, consultants, teaching professionals, researchers, and academic theorists have long recognized the importance of strategic choice alignment as a determinant of firm performance and as a measure of strategic leadership competence. But how do we know which organizational elements need to be aligned? How do we determine if those elements are aligned or misaligned, and how do we prioritize misalignments which most urgently need to be corrected? This paper proposes a ten-element best-fit alignment stack of strategic choice meta-frameworks drawn from the author's experience as both a business leader and graduate school professor and offers a simple assessment mechanism that identifies the most critical misalignments in a firm.
\end{abstract}

\section{Keywords}

Strategy, Leadership, Decision-Making, Organizational Analysis, Alignment, Performance

\section{Introduction}

The correct answer in an MBA classroom to nearly any question is: "it depends". It depends on the industry sector(s) in which the firm participates, i.e., its market position, its strategies, its legal structure, its financial circumstances, its leadership expectations, its risk tolerance, its mission, its vision, its goals, and its very notion of effectiveness.

There is no single recipe for business success, no one strategic choice which will determine firm performance. Rather it is the alignment of multiple interdependent strategic choices that creates the environment in which tactical and operational initiatives can lead to excellent firm performance.

Graduate school is about teaching the application of frameworks, not the 
memorization of recipes; we teach our MBA students principles, theories, and ideas that help them understand complex situations that present with ambiguities, uncertainties, and undetermined numbers of interdependent probabilities.

The tendency of under-led organizations to devolve into self-interested factions has not been erased by 21 st-century technologies and multi-cultural influences; it has been accelerated and intensified. Factional conflict-the unmanaged formation of unsanctioned organizational sub-units fueled by alienation and mistrust-is a greater threat than ever before.

And so the need for creating alignment, for getting our organizational segments and strategic initiatives "on the same page", is more crucial than ever before. But what is it that needs to be aligned? Which organizational characteristics are strategic choices, versus tactical or operational necessities? How do we know if our organizations are aligned or misaligned and how do we prioritize misalignments we might detect?

This paper proposes a framework and approach to assessing alignment of strategic choices that have been developed over decades of organizational profiling in the private sector-analyzing the organizations of competitors, customers, suppliers, teaming partners, acquisition candidates, and subsidiary holdings of a conglomerate firm. It was incorporated into the Leadership Development program developed at the author's firm and refined as it was brought into academia and taught in MBA courses at Athabasca University and University of Maryland University College Europe in recent years.

There have been hundreds of outstanding papers written on the subject of alignment of various pairs of organizational elements: strategy and structure, leadership and decision-making, power mode and motivation, etc. This paper will propose a more ambitious synthesized framework; creating four proposed alignment "stacks" made up of ten elemental typologies each.

It will conclude with the presentation of a simple scoring tool for assessing alignment and misalignment using these stacked typologies and for identifying the specific element pairings of an organization where misalignment is most likely to be causing severe negative impacts-those element pairings whose alignment score equals five. The "fives" are likely to be the source(s) of deeply seated factional conflict within the firm.

\section{Methodology}

The Elaboration Theory of Instruction [1] describes a process of formative research which uses multiple naturalistic cases [2] and synthesis of previously accepted theoretical frameworks to develop expanded or adapted theoretical frameworks that enrich learning. This paper has been developed according to those principles.

The development of this paper's theorized alignment "stack" draws from existing analytical frameworks in a triangulation that arose from the author's reflections upon three different sets of experiences and collections of post facto 
cases.

The first is a 38-year business career during which hundreds of organizational profiles were developed and/or evaluated for competitors, customers, suppliers, acquisition candidates, and potential teaming partners. The second is several years of teaching and course-writing in Athabasca University's MBA program, particularly the STOA603 Strategy and Organizational Analysis course and the STCH600 Making Strategic Decisions course. The third is this past year of teaching multiple sections of courses in University of Maryland University College Europe's MBA program-MBA610 Leading People and Organizations, AMBA 670 Managing Strategy In The Global Marketplace, and MBA670 Strategic Decision-making.

Between corporate organizational profile assessments, student discussions online in the classroom, and student papers which apply the frameworks and theories which have been assembled into this synthesis, several hundred case studies focused on the subject of alignment of organizational elements and strategic choices have been considered, spanning all major industry segments of private sector firms, public sector agencies, NGO's charities and other non-profits, foreign firms, educational institutions, and military units.

The idea of "scored" stack of four-quadrant typologies built on the foundation of Quinn and Rohrbaugh's CVF foundation [3] arose from the series and sequence of lessons in the syllabus of Athabasca University's STOA603 Strategy and Organizational Analysis course. While the course was not written to be taught with a theme of sequential alignment assessment, I recognized a strong similarity in the sequence of the elements included in the weekly lesson plans to the sequence of the elements we required in our corporate profiling function during my executive career.

Early "prototypes" of the alignment stack table were developed as supplements to stimulate discussion and draw out a clearer understanding of the CVF and its practical application; at first clusters of 2 or 3 elements, gradually adding elements until the current 10-element stack emerged. Different means of "scoring" alignments and misalignments were discussed as a pedagogical device, i.e. to focus the students on the practical considerations of prioritizing strategic decision-making in the real world.

While most of the specific elements have been assigned to a four-quadrant framework from one author, there are exceptions, i.e. four typologies selected from six in the original paper, or ideas of two authors combined in one element. The overarching purpose is to provide four suitable matches for the four Quinn [3] effectiveness models in each table element, not to select typologies merely to fit an arbitrary quadrant typology format.

While not planned as an element in a research project per se, the feedback, criticism, and recommendations of over 200 mid-career graduate business students using this diagnostic tool to assess their own firms have driven the revisions and refinements that have led to the proposed alignment stack table and 
scorecard as it is currently constructed.

With the last three sections of UMUC's MBA670 classes having completed an in-classroom application exercise of the alignment stack and "rule of 5" to their own organizations without any recommendations for modification, the idea was considered to be suitable for publication, consideration, and refinement by a wider community of academics and practitioners.

\section{The Theorized Alignment Stack}

The ten elements included in the synthesized alignment framework have been selected from the author's four decades of experience as a business executive, graduate business course writer, MBA professor, and Leadership Development program director. The elements and lead authors of those previously accepted frameworks are:

- notional effectiveness-Quinn [3]

- strategy-Porter [4]

- structure-Mintzberg [5]

- culture-Goffee [6]

- decision-making-Lewin [7]

- leadership-Bass [8]

- motivation-various

- power-mode-French-Raven [9]

- conflict resolution mode-Blake-Moulton [10]

- change mode-Kotter, Beer [11] [12]

Each will be briefly described in a subsequent section. The paper assumes the readers' passing acquaintance with these widely-cited authors' seminal works. It would take hundreds of pages to explain them adequately to a neophyte-whole courses have been built around some of them.

As a practical matter, organizational analysis using these ten elements is best done in sequence. Every decision in the firm is taken to make it more effective, so the first thing to learn about it is its notion of effectiveness; structure is selected to support strategy, decision-making preferences will influence the type of leaders who are selected, and so on. Each strategic choice will inform-not dictate, but inform-subsequent strategic choices, so the key to quickly reaching an accurate understanding of the entire firm is to approach the task of profiling the correct sequence. Proper sequencing will also make misalignments more readily apparent.

The foundation for alignment is the Competing Values Framework (CVF) developed by Quinn and Rohrbaugh [3]. It is the most fundamental strategic choice the firm will undertake; all others are informed by this first choice, and it will be the basis against which alignment is mapped. A firm's dominant notional effectiveness model is the most crucial characteristic of the firm to profile correctly; like the foundation of a building, our understanding of notional effectiveness must be solid and sound or subsequent determinations about firm cha- 
racteristics will be unstable and unreliable.

\subsection{Effectiveness}

The first thing to understand about any organization its notion of effectiveness, because every strategic decision made in an organization is taken to make the organization more effective; however, there is no single notional definition of effectiveness, no one criteria that everyone agrees correctly measures the effectiveness of a firm or its strategic choices. Decisions that appear foolish from our own notional perspectives of effectiveness may be quite sensible within the framework of the firm's effectiveness model. It is incumbent on the analyst to understand the firm, not to project his/her preferences and biases onto it.

Quinn and Rohrbaugh [3] organized 95 indicators of effectiveness into a quadrant framework with whose two-dimensional criteria are internal versus external focus along its $x$-axis, and flexibility versus control as its $y$-axis. A graphic depiction is provided as Appendix A. The result is four competing effectiveness models-dominant conceptions of the term that inform the organization's strategic decisions. Quinn [13] went on to apply the idea of CVF to individual leaders; our individual frames of reference can be mapped to the same quadrant as can our collective associations we call firms.

When we teach CVF in graduate school we will often explain the internal/external values of $\mathrm{x}$-axis as the firm's reflexive source of validation and the flexibility/control values of the $y$-axis as its instinctive tradeoff preference. If a customer survey (external) indicated a firm was very effective, but an employee survey (internal) indicated a firm was very ineffective, which would be instinctively actionable? And will the firm give up control to achieve greater flexibility, or will it give up flexibility to increase control? We use the term "emphasis" to indicate dominant, but not exclusive modality.

If there is no dominant effectiveness model established for the firm, then internal factions will arise around the four competing values, and that factional loyalty will be more powerful than the firm in terms of individual's membership identity. Factional conflict is most severe and damaging between opposing diagonals on the CVF quadrant, where both axes are in conflict. This intensified conflict between diagonals applies to individuals as well as factions; conflicting notions of effectiveness is the underlying cause of many, perhaps most, destructive interpersonal conflicts between leaders in the firm. That idea of diagonal conflict being the most severe is the basis of the "rule of 5 " explained later in the paper.

The first quadrant (internal, flexibility) is labeled Human Relations (HR). The HR quadrant defines effectiveness as cohesion, morale, a sense of belonging, and personal development within a collective tribal framework. Its shorthand generic slogan is "doing things together"; that is what the HR firm values above all else. Think of Pixar in the Jobs era.

The second quadrant (internal, control) is labeled Internal Process (IP). The 
IP quadrant defines effectiveness as stability and reliability through compliance with internal rules, and avoidance of deviation. Its shorthand generic slogan is "doing things the right way"; that is what the IP firm values above all else. Think of Inbev.

The third quadrant (external, flexibility) is labeled Open Systems (OS). The OS quadrant defines effectiveness as rapid growth, resource acquisition, innovation, readiness to change, and external networking. Its shorthand generic slogan is "doing different things"; that is what the OS firms values above all else. Think of Tesla.

The fourth quadrant (external, control) is labeled Rational Goal (RG). The RG quadrant defines effectiveness as productivity and efficiency through planning and goal-setting, and market benchmarking. Its shorthand generic slogan is "doing things better"; beating competitive benchmarks is what the RG firm values above all else. Think of GE in the Welch era.

\subsection{Strategy}

Hundreds of books and thousands of journal articles have been written on the subject of business strategy. Nearly all of them in recent decades will reference Michael Porter's generic strategies [4] Porter provides the framework for understanding strategy choices by first selecting one of four generic strategies for the firm-low-cost provider, differentiator, focused differentiator, or niche.

The low-cost provider is pretty straightforward, the firm's competitive advantage is the lowest cost-acquisition or ownership or both. The differentiator selects features and characteristics in products and services that differentiate them and create value in the minds of customers. The focused differentiator will seek to differentiate offerings to appeal to particular markets, needs, or customer groups. Niche strategies are the extremely selective positioning choices and unique business propositions that defy characterization.

\subsection{Structure}

Henry Mintzberg is certainly among the most influential thinkers in the field of organizational behavior in any era. He classified organizational structures into four types [5]: professional, machine, entrepreneurial, and hybrid.

Broadly thumbnailed, the professional structure is built around the professional skills/certifications that firm members bring to the firm. The machine structure is built around defined tasks. The entrepreneurial structure is loose and temporal, intentionally vague. The hybrid structure is a synthesis of the other three.

\subsection{Culture}

Of the ten organizational elements selected for the alignment table, culture is probably the one most extensively written about and the least understood. I attended a lecture once where one of Canada's foremost experts on organizational 
culture began by stating that culture is hard to define, and then spent 90 minutes proving it. Every newly hired coach or CEO proclaims the need to "change the culture", which will be the first priority of their successor, and their successor, and so on.

The problem with defining culture is that we are dealing with the white space, not the black ink. The "about us" tab on a corporate website will tell us something about leadership's aspirations for the firm, but culture is the way things really work; it is the informal org chart and the unwritten rules and the things that draw praise and scorn from peers. In my MBA courses, I define one edge of culture as the boundary between the tolerated and the intolerable. Goffee and Jones [6] provided a quadrant typology for cultures with labels that resonate: communal, fragmented, mercenary, and networked.

\subsection{Decision-Making}

Some things can't be improved upon. Lewin's [7] typology of decision-making styles has survived three generations and the transition from the industrial age to the information age to the current state of flux. The four archetypes: authoritarian, consensus, collaborative, and laissez-faire have adequately described the dominant decision-making modes of organizations big and small, and have been applied to thousands of firms over millions of student assignments in business courses around the world.

\subsection{Leadership}

Bass [8] provided a workable distinction between transactional and transformational leadership, and his frameworks were robust enough to accommodate the refinements of adding situation and servant leadership that were developed by various successive researchers and leadership theorists.

Leaders come in all varieties and the many facets of leadership have been the subject of observation and study since ancient times. But just as effectiveness is the foundation of understanding organizational behavior, leadership type and orientation is the foundation of developing authentic leadership and the key to aligning leadership with the needs of the led.

\subsection{Motivation}

The literature covering motivation spans business, economics, sociology, psychology, theology, philosophy, and history. Universal is the general classification of motivations into the categories of extrinsic, intrinsic, peer, and personal. Firms will employ some mixture of all four types at one point or another, but again we are seeking to understand the dominant choice, the go-to default setting that is almost instinctive in nature.

\subsection{Power Mode}

The oldest of our selected quadratic frameworks is "The Basis of Social Power" 
[9] which like Lewin [7] and Blake-Moulton [10] has served to clarify and stratify subsequent research and discovery into an organized and actionable ody of knowledge that practitioners have found useful for decades.

Referent, position, reward, expert-these four categories of power describe the range of power-application employed in the firm. While all will be used when circumstances dictate, one will be the dominant power mode and those who learn to accumulate and dispatch power within the preferred mode of the firm will increase their stature and standing in the firm. Private enterprise is a voluntary association of individuals, so all power is applied in any of the categories by means of persuasion. Understanding power-mode in the firm involves determining which of the four forms is accepted as the most persuasive.

\subsection{Conflict Resolution Mode}

Organizational Behavior (OB) researchers have been interested in conflict and conflict resolution for as long as organizations have been systematically studied. A durable framework for categorizing conflict resolution styles is Blake and Moulton's framework [10] which proposed four typologies: accommodating, consensus, compromise, competitive

\subsection{Change Mode}

Few organizations truly master change but many have accepted the reality that with globalization and technology, change is now the steady-state, occasionally interrupted by brief interludes of status quo. Beer and Nohria identified Theory $E$ and Theory $O$, the two instigating forces of major change [13]. Kotter's 8-step change process remains the gold standard for leading major change [11], and the broad category of continuous improvement makes up the fourth of the change modes selected in this paper for consideration of alignment.

In the following sections, the paper will briefly discuss the proposed best-fit frameworks or typologies for each of the four Quinn and Rohrbaugh CVF effectiveness [3] quadrants. The combined alignment stack table is found in Appen$\operatorname{dix} B$.

\section{Best-Fit Stack-Human Relations (HR) Quadrant}

The HR effectiveness model values strong tribal identity, cohesion, and a sense of belonging [3]. It is defined by an internal validation focus and a priority of flexibility over control that creates a unique membership experience.

A differentiation strategy [4] is proposed as a best-fit for this quadrant. Differentiated products and services are compatible with a unique and differentiated membership employment experience. Famously differentiated brands like Harley-Davidson, BMW, and Westin often reflect the "membership" notion of effectiveness in the firms which created them.

A professional structure [5] provides the flexibility for individual growth and development, a key characteristic of the HR quadrant firms. The communal culture [6] is a synonymous label for the HR quadrant; a culture of community is 
compatible with an effectiveness notion of cohesiveness. Consensus decision-making [7] is typical of tribal communities, including those assembled for profit-making. Servant Leadership [14] is desirable in the HR quadrant where cohesion and belonging are paramount.

HR quadrant firms' overarching goals of cohesion, belonging, and community make peer motivation a good choice. Group rewards and participatory distribution processes fit this category as does peer pressure - i.e. people motivated to behave according to tribal norms to be accepted and retain membership standing. Referent power [9] fits the communal sensibilities of HR quadrant firms, its distinguishing fluidity a match for the flexibility dimension on the CVF grid. Accommodating conflict mode [10] supports the values of cohesion and membership and provides for flexibility in application. Beer's Theory $\mathrm{O}$ change [12] is the final selection for best-fit elements; its focus on developing new organizational capabilities, rather than specific outcomes, makes it a compatible choice for the HR model firm.

\section{Best-Fit Stack-Internal Process (IP) Quadrant}

The IP effectiveness model values compliance with internal procedures and policies and predictability [3]. It is defined by an internal validation focus and a priority of control over flexibility that creates an environment of reliable and repeatable workflows.

A low-cost-provider strategy [4] is proposed as a best-fit for this quadrant. Costs are minimized by standardization, elimination of waste, economies of scale, and the absence of deviation. Incremental changes to processes that lower unit costs can be predictably implemented in an organization where compliance is the first principle. Internal best-practices can be replicated quickly in a process-based environment.

A machine structure [5] provides the environment for process compliance to flourish in the IP firm. The fragmented culture [6] eliminates the distractions of group loyalties or creative thinking, and pins employment to process explicitly. Authoritarian decision-making [7] ensures adherence to process requirements. Situational leadership [8] is a best-fit blend of maintenance of the status quo and process change in a prescribed and orderly manner.

IP quadrant firms' overarching goal of compliance makes extrinsic motivation a good choice. Punishment for non-compliance, more than incentives for expected compliance, keeps the priority on process control. Position power [9] fits the rigid allegiance to the hierarchy of the IP quadrant firms. Compromise conflict mode [10] is the least disruptive, minimizes risk and ensures change is incremental. Continuous Process Improvement (CPI) is the obvious choice for change mode.

\section{Best-Fit Stack-Open Systems (OS) Quadrant}

The OS effectiveness model values rapid growth resource acquisition, and inno- 
vation [3]. It is defined by an external validation focus and a priority of flexibility over control that removes barriers to innovation and opportunistic expansion.

A focused differentiation strategy [4] is proposed as a best-fit for this quadrant, although others could be successfully employed. The advantage of focused differentiation for the OS quadrant is that its entrepreneurial bent will tend to lead it into multiple unrelated ventures in unpredictable time-frames. A generic focused differentiation strategy provides for rapid adaption and cross-over to new markets and competitive positions. A firm skilled in focused differentiation can manage several different focused differentiation strategies in several different business segments at the same time. And new strategies to meet new opportunities can be assembled quickly by borrowing elements of existing business strategies and rolling them into new applications.

The entrepreneurial structure [5] is an easy choice for the entrepreneurial OS firm. The networked culture [6] and collaborative decision-making mode [7] serve this quadrant's penchant for external teaming and partnering well. Transformational leadership [8] makes sense since the Open Systems firms are continuously reinventing themselves.

OS quadrant firms' overarching goals of growth and innovation intrinsic motivation a good choice-the joy of invention motivates inventors. Expert power [9] is a natural consequence of the absence of structure in the ad-hoc frenzy of the Open System firm, more flock than hive, prowess is the currency of power, as opposed to titles or seniority. Consensus conflict mode [10] fills the vacuum created by the lack of structure typical in OS firms, and Kotter's 8-step [11] is the preferred change mode where major change is the constant and status quo is the brief interlude.

\section{Best-Fit Stack一Rational Goal (RG) Quadrant}

The RG effectiveness model values productivity and efficiency [3]. It is defined by an external validation focus and a priority of control over flexibility that is achieved through planning, goal-setting, and cyclical measurement of progress against benchmarks.

Several of the generic strategies [4] could be executed successfully in this quadrant, but niche is proposed as a best-fit. The concept of niche - the intersection of product feature differentiation and market segmentation around need - is compatible with the RG firm's love of definition and specific goal-seeking to plan. Disciplined consumer products companies will have plans and goals broken down by product family, band, product line, and individual products for each managerial region and district. Each could be considered a niche, as planning and goal setting recognizes the unique market circumstances for each planning/execution cycle.

The hybrid structure [5] is the best-fit choice for the productivity-driven RG firm, with market-specific customer-facing functions supported by common back-office functions. The mercenary culture [6] fits the hit-your-numbers 
demands of the RG firm, and laissez-faire [7] is a good decision-making mode when outcomes, not process, are the bottom line. "Do whatever it takes" is the mantra of a quadrant with decisions pushed out as close to the marketplace as possible. Just hit your numbers. Transactional leadership [8] and personal motivation (exceeding expectations on evaluative measures) support goal-seeking activities well.

RG quadrant firms' commonly employ rewards [9] as their favored power mode-bonuses, commissions, pay increases, promotions. Competitive conflict mode [10] is efficient, a highly sought value of the RG firm. Two alternative ideas are presented, and one is chosen—no wasted motion. Beer's [12] Theory E change mode is a best-fit for the RG quadrant; it selects economic goals first and then uses goal attainment to drive change through the organization. In a quadrant where productivity is king and meeting planned numeric objectives is prized Theory E is just the ticket.

\section{Assessing Alignment Using the Scorecard}

Assessing alignment and misalignment is fairly straightforward using the alignment stack table provided as Appendix B and the alignment scorecard for recording assessment results provided in Appendix C. The exercise can be done by an individual or by separate independently working individuals or by group consensus.

Note that the table should be approached "bottoms up" working from the CVF effectiveness model [3] selection upward. It is presented that way to support the idea of foundations in organizational construction-each choice informing the ones to follow. MBA students have learned and applied CVF since it was introduced [15] and studied characteristics of firms using the framework. We now build upon that critical analysis to provide a structured means of analyzing alignment with the effectiveness model selected for the firm.

The elements selected for analysis and the typologies and academic frameworks selected to categorize them have been the subject of hundreds, perhaps thousands of journal articles. This paper does not propose a specific method for assigning any one of the typologies to the subject firm; that process can be whatever method or combinations of methods the analyst prefers. For academics and practitioners familiar with the literature upon which this alignment framework is based and familiar with the subject organization, a "first to mind" word choice is often the best.

What we are looking for is a sense of strategic alignment where element selected is from the same column stack as the effectiveness model, or misalignment where element and effectiveness are not found in the same stack, or severe misalignment where the sum of the two misaligned column numbers equals five.

This exercise has been done as a classroom assignment in my MBA capstone course (Strategic Decision-Making) at University of Maryland University College-Europe. The students have studied all of the elements of organizational 
design and analysis in prior coursework and are mostly familiar with the classic frameworks used in constructing this synthesis. I have them use their own organizations as the subject, so they do not need to do extensive research to form their opinions and make their choices. Dozens of participating students have considered private sector firms (American and German), government agencies, NGO's and charities, and military units.

We step through the elements one at a time, starting with the effectiveness model selection. Typically, I will read the four choices aloud, provide some descriptive keywords to jog students' memories of the defining characteristics of the archetypes, and then allow $1-2$ minutes to consider the elements and make a selection.

When we have completed the assessment of the ten individual elements we count up the numbers of elements in alignment, the numbers in misalignment, and the numbers in severe misalignment (columns sum to five). And then we discuss our findings, their implications, the usefulness of framework as a diagnostic tool, and any insights gained during the exercise.

\section{Analyzing the Alignment Scores}

Few firms will exhibit perfect alignment-i.e. a perfect row of identical column number scores. Most well-functioning organizations will have one or two elements out of the proposed best-fit alignment, and often the results of intentional strategic decisions. Some firms will look like a scatter-gram, with elements from all four columns distributed with no rhyme or reason. When we discuss those organizations it is no surprise that "chaos" is the word that comes up most frequently to describe the firm's performance. Organizations evolve, and often the firms or organizational units that exhibit near-randomness and rampant misalignment have been assembled from fragments of legacy organizations that have never been systematically integrated.

It is not the number of misalignments that are most noteworthy, it is the number whose sum equals five. Those are the severe misalignments often cause disruption, conflict, and severely diminished performance. A single sum-score of five may be attributed to a specific change or some other traumatic event in firm history, but firms which produce more than two sum scores of 5 will typically be severely dysfunctional and in a state of extreme distress-facing bankruptcy, merger into another organization, de-funding, or some other existential threat.

The significance of a sum score of 5 goes back to the Quinn and Rohrbaugh CVF quadrant framework [3] and the two dimensions upon which notions of effectiveness rest. Column sums of 3, 4, 6, and 7 are produced by misalignment from adjacent quadrants who share one of the two differentiating dimensions either internal/external focus or flexibility/control priority. But column sums of $5(2+3$ or $1+4)$ are diagonals on the CVF quadrant grid, so opposing beliefs about effectiveness exist along both dimensions. These are often bridges too far, and the best-fit elements for each effectiveness model tend to follow the pattern 
of diagonal severity, in my experience.

When you disagree with me on one dimension, you may question my intelligence, but when you disagree with me on both dimensions you start to question my motivation and my moral compass. I think that you are trying to destroy the firm because from my frame of reference you are doing things that make us less effective. And it is indeed intentional; you are doing what you think will make the firm more effective, from your perspective of what effectiveness means. At the diagonals, we have diametrically opposed beliefs about what effectiveness means and what needs to be done to improve it. The higher we go up the organizational chart, the deeper those beliefs become embedded and the more passionate we become about advocating/defending them.

We can see this all around us, and not just in our businesses; it is why we fight. My own personal quadrant is Open Systems, and it is no accident that my career flourished in firms firmly rooted in that frame of reference. I don't just become frustrated at the rules and permissions enacted by Internal Process factions, organizations, and leaders, I attack them, because I truly believe they are destroying value. I'm certain that my IP friends are just as adamant that my wanton disregard for rules is an existential threat. We only fight because we care.

If we did not care, we would retreat to our own factions and sulk. In my experience as an executive that is the worst possible circumstance - deeply held factional hostility that is no longer worth discussing to the combatants, who retreat to their echo chambers and pretend to be on the same team. Keyword pretend. Those passive-aggressive factional silent stand-offs are toxic to any organization, but those engaged will deny there is a civil war going on. We often misdiagnose them as personality conflicts or disputes over this policy or that, when there is a much deeper problem.

But factional conflicts caused by strategic misalignment will reveal itself and its root cause as sums of 5 in our stack of elements when we go through the process of analysis in sequence. Our factional conflicts will leap off the page, and then we can address them, correct the misalignment, and improve organizational performance with a sense of urgency.

\section{Conclusions and Recommendations}

The proposed alignment stack table creates a systematic method for organizational profiling of firms. There are several useful applications for using such a tool that come readily to mind:

- assessing the compatibility of potential M\&A targets and estimating integration plan complexities

- identifying exploitable weaknesses of competitors

- strengthening alliances with customers in areas of strategic compatibilities

- prioritizing strategic changes needed to improve the performance of the firm

- periodically internal canvassing of business units to pro-actively identify problems requiring attention 
- creating a unified perspective of strategic decision-making to develop leaders and strategists

By identifying the elements most severely misaligned with the firms' notions of effectiveness-those with a stack number sum of five-executives can prioritize their resources on bringing those elements into alignment that will have the greatest impact on firm performance.

The paper's proposed synthesis of existing frameworks is theoretical, but it has been thought-tested and assessed favorably in graduate school classes whose students are typically middle to upper-level managers with 10 - 15 years of experience at the back end of their MBA program; they do not possess skills, knowledge, and capabilities beyond those we would expect to find in the senior management ranks of most firms. The risk of the idea being an irrelevant academic abstraction too complex for practical use is low.

Additional research and more widespread application should be pursued to validate the selection of the ten elements, the selection, and application of the typologies found in the literature for each, and the best-fit placement of typologies in each of the stacks. A rigorous vetting of the conceptual framework constructed and a consensus on methodologies by which it can be applied would strengthen its credibility and viability as a diagnostic tool for practitioners and academics alike.

\section{Conflicts of Interest}

The author declares no conflicts of interest regarding the publication of this paper.

\section{References}

[1] Reigeluth, C.M. and Stein, F.S. (1983) The Elaboration Theory of Instruction. In: Reigeluth, C.M., Ed., Instructional-Design Theories and Models. An Overview of Their Current Status, Lawrence Erlbaum Associates, Hillsdale, NJ, 335-382.

[2] Yin, R.K. (1984) Case Study Research Design and Methods. Sage Publications, Beverly Hills, CA.

[3] Quinn, R.E. and Rohrbaugh, J. (1983) A Spatial Model of Effectiveness Criteria: Towards a Competing Values Approach to Organizational Analysis. Management Science, 29, 363-377. https://doi.org/10.1287/mnsc.29.3.363

[4] Porter, M.E. (1980) Competitive Strategy: Techniques for Analyzing Industries and Competitors. The Free Press, New York.

[5] Lundberg, C. and Mintzberg, H. (1991) Mintzberg on Management: Inside Our Strange World of Organizations. Industrial and Labor Relations Review, 44, 771-772. https://doi.org/10.2307/2524475

[6] Goffee, R. and Jones, G. (1996) What Holds the Modern Company Together? Harvard Business Review, 74, 133-148.

[7] Lewin, A.Y. and Shakun, M.F. (1976) Situational Normativism: A Descriptive-Normative Approach to Decision Making and Policy Sciences. Policy Sciences, 7, 1-10. https://doi.org/10.1007/BF00146017

[8] Bass, B.M. (1985) Leadership and Performance beyond Expectations. Free Press, New York. 
[9] French, J.J. and Raven, B. (1959) The Bases of Social Power. In: Cartwright, D. and Cartwright, D., Eds., Studies in Social Power, Oxford, England, 150-167.

[10] Blake, R.R. and Mouton, J.S. (1972) The 1,9 Managerial Style. In: Managerial Grid, Gulf Publishing, Houston, 57-84.

[11] Kotter, J. (1997) Leading Change: A Conversation with John P. Kotter. Strategy \& Leadership, 25, 18-23. https://doi.org/10.1108/eb054576

[12] Beer, M., \& Nohria, N. (2009). Cracking the Code of Change. The Principles and Practice of Change, 63-73. https://doi.org/10.1007/978-1-137-16511-4_4

[13] Quinn, R.E. (1984) Applying the Competing Values Approach to Leadership: Toward an Integrative Framework. Leaders and Managers, 10-27. https://doi.org/10.1016/B978-0-08-030943-9.50010-3

[14] Greenleaf, R. (1991) The Servant as Leader ([Rev. ed.). Robert K. Greenleaf Center, Indianapolis, IN.

[15] Thompson, M.P. (1993) Using the Competing Values Framework in the Classroom. Human Resource Management, 32, 101-119.

https://doi.org/10.1002/hrm.3930320106 
Appendix A: CVF Graphic

EFFECTIVENESS EMPHASIS

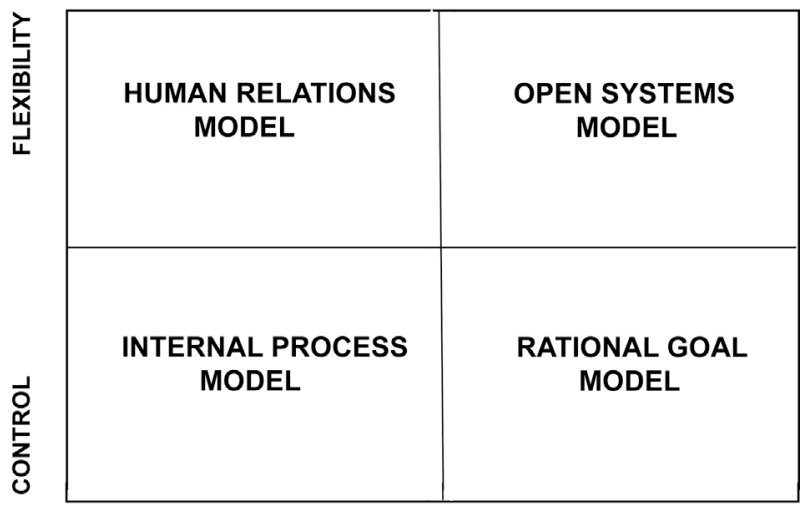

INTERNAL

EXTERNAL

\section{COMPETING VALUES FRAMEWORK}

\section{Appendix B: Alignment Stack}

ELEMENT ALIGNMENT STACK: BEST-FIT TYPOLOGIES

\begin{tabular}{|l|l|c|c|c|c|}
\hline & & Do It Together & Do It Right & Do It Different & Do It Better \\
\hline CHANGE MODE & Kotter (1991)/Beers (2009) & Theory O & Process Improvement & Kotter 8-Step & Theory E \\
\hline CONFLICT & Blake-Moulton (1972) & Accommodating & Compromise & Consensus & Competitive \\
\hline POWER MODE & French-Raven (1959) & Referent & Position & Expert & Reward \\
\hline MOTIVATION & various & Peer & Extrinsic & Intrinsic & Personal \\
\hline LEADERSHIP & Bass (1985) Greenleaf (1991) & Servant & Situational & Transformational & Transactional \\
\hline DECISION & Lewin (1976) & Consensus & Authoritarian & Collaborative & Laissez Faire \\
\hline CULTURE & Goffee-Jones (1996) & Communal & Fragmented & Networked & Mercenary \\
\hline STRUCTURE & Mintzberg (1991) & Professional & Machine & Entrepreneurial & Hybrid \\
\hline STRATEGY & Porter (1980) & Differentiation & Low-cost Provider & Focused Differentiation & Niche \\
\hline & & & & & \\
\hline PERFORMANCE & & "Do It Together" & "Do It Right" & "Do It Different" & "Do It Better" \\
\hline PROBLEM SOLVING & & Train & Add Rule & Change Role & Add Goal \\
\hline PRIORITY & & Cohesion & Compliance & Growth & Predictability \\
\hline EFFECTIVENESS & Quinn (1983) & Human relations & Internal process & Open systems & Rational Goal \\
\hline & & $\mathbf{1}$ & $\mathbf{2}$ & $\mathbf{3}$ & $\mathbf{4}$ \\
\hline
\end{tabular}

\section{Appendix C: Alignment Scorecard}

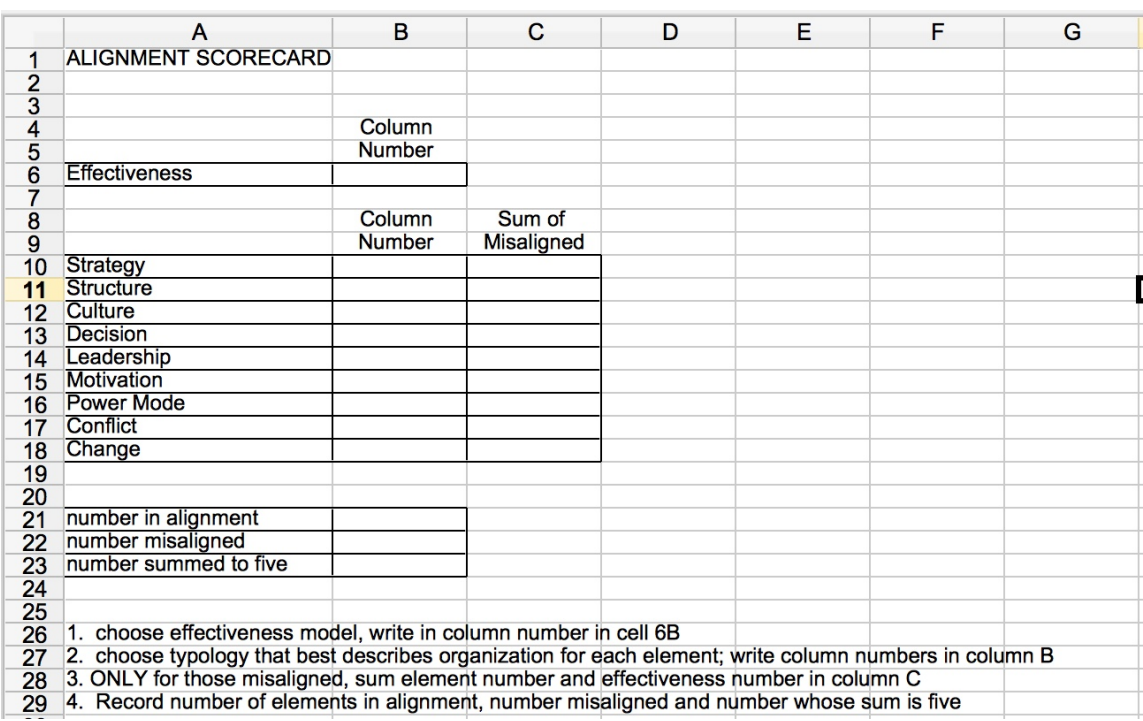

